\title{
Recognition of Emotion From Facial Expression for Autism Disorder
}

\author{
A. Sivasangari, Bhanu Prakash.V, G.V.V.Rajesh
}

\begin{abstract}
Facial expressions convey verbal indications that play an important role in interpersonal relationships. Despite the fact that people immediately perceive facial expressions for all intents and purposes, solid expression recognition by machine is still a challenge. From the point of view of automatic recognition, The facial expression may included the figurations of the facial parts and their spatial relationships or changes in the pigmentation of the face. The study of automatic facial recognition addresses issues relating to the static or dynamic qualities of such distortion or facial pigmentation. Use The Camera to capture the live images of autism people.

Index Terms - Augmentation, Deep convolutional neural network, deep learning, Live facial images, Camera, OpenCV, Tensorflow.
\end{abstract}

\section{INTRODUCTION}

Facial expression is a important of nonverbal communication. We can use Facial expressions recognition systems in many fields, like criminal interrogation, psychiatry etc. We can find main basic facial expressions such as Anger, Disgust, Happiness, Fear, Etc. We can find the emotions of Autism Disorder patients with the help of their Facial Expressions, the autism disorder patients are not having control over their emotions and actions. Through facial expressions we can find the emotions and help them to control over their actions. We can use Automatic facial expression recognition helps to monitoring the patient behavior [8], In this we are going to find out the Facial Expressions of the Autism Disorder By placing the people in front of the camera and we monitor the facial expressions continuously with the help of the expressions we find predicted the emotions of the person $[9,10]$.

\section{RELATED WORK}

In literature, several techniques for facial expression recognition have been proposed, which include SVM classifiers which is used to classify different facial expressions, Venna mayya[1] Proposed Deep conventional neural network (DCNN) by using this model we train our model with deep neural network which helps to find the facial expressions with high accuracy but we must find the Variables to classify deeply to get better output, Pawel Tharnowski [2] proposed the KNN and MLP classifier which classify the data into different expressions and train

Dr. A. Sivasangari, Department of Information Technology, Sathyabama Institute of Science and Technology, Chennai-T.N, India. (Email: sivasangarikavya@gmail.com)

Bhanu prakash.V, Information Technology Sathyabama Institute of Science and Technology, Chennai, T.N, India. (E-mail: bannysholai@gmail.com)

G.V.V.Rajesh, Information Technology Sathyabama Institute of Science and Technology, Chennai, T.N, India. (E-mail: rajesh22b4u@gmail.com)
Revised Manuscript Received on July 10, 2019.

the model with the help of the classifier algorithms when we run the model on the real outputs they classify the images buy using their trained inputs and give the output according to their predictions. Murthy.G.R.S [3] Proposed to use Eigenvectors and Eigenfaces first we divided the data images into six main facial expressions and then we calculate the Eigen-distance between the points and we calculate every test image individually and we develop the model and we run the model to use for finding the different expressions. Their having lot of models used to find the Facial expressions. Aliaa A.A Youssif[4] proposed Automatic Facial expression recognition system, In this he first detect the face and extract facial features and take the geometric features and run by using OpenCv, This model helps to run the facial expression detection model live so we can use this model anywhere. Philipp Michel[5] proposed Real time facial expression recognition by using SVM algorithm in this first we train the model by using svm training and then it extract features with the svm classifier and get the output in the live video stream. Erhan, D[6] proposed the scalable object detection using deep neural network which helps to find the live object detection by using deep networks, It is hard to run live object detection because the frame rate and the refresh rate of the system are very high we need top end model device to run this program but by using scalable model we can run on any machine for its compact ability . Zhang[7] Proposed Facial expression recognition using facial movement features where we use the facial features and the movements we use nose, eyes and cheeks movements and train the model according to it and classify the model according to it.

\section{PROPOSED WORK}

\section{A. Deep Convolutional Neural Network(DCNN)}

A Deep Convolutional Neural Network (DCNN) is the proposed architecture used for the Data set (Cohnkandae (CK and $\mathrm{CK}+$ ) database) it takes the data present in the data set and perform deep neural network we classify the dataset into different emotions and with the help of the classification we train the model using deep convolutional neural network where the data is classified deeper and give better results. The DCNN is a very powerful algorithm compare to SVM and help to get much accuracy compare then other database. The data is much classified it go very deep and classified. 


\section{B.Data Classification}

The Cohn-kandae(CK and $\mathrm{CK}+$ ) database contain above 1000 variety pics of facial expressions from different subjects. JAFFE(Japaneses Female Facial Expressions) in this dataset it contains various facial expressions of Different female subjects, SFEW(The Static Facial Expression in the wild) This dataset also contains various facial expressions of the different subjects, In all the three datasets the $\mathrm{CK}+$ dataset contains more classified image database so we are using $\mathrm{CK}+$ dataset to train our model, These pics are classified into different emotions folders and this folders named according to the name of the emotion and this classified data is used for training purpose. The data are train according to their emotions we need to train the model with more examples so that the model give better accurate output.In the data classification we divided the expressions based on the individual facial characteristics (eyes, nose, mouth and face), The following table shows the number of images of each expression in different datasets.Anger(An),Disgust(Di),Fear(Fe)

Table 1: Number of images for each Expression in Datasets

\begin{tabular}{|l|l|l|l|l|l|l|}
\hline & An & Di & Fe & $\mathrm{Ha}$ & $\mathrm{Ne}$ & $\mathrm{Sa}$ \\
\hline CK+ & 360 & 275 & 160 & 389 & 130 & 256 \\
\hline JAFEE & 30 & 26 & 12 & 34 & 22 & 19 \\
\hline SFEW & 110 & 79 & 88 & 120 & 78 & 97 \\
\hline
\end{tabular}

Model Training

Cohn-kandae(CK and $\mathrm{CK}+$ ) dataset is used for data training the model by use Deep convolutional Neural network the DCNN is very powerful algorithm in this algorithm we divided the data set into deep neural layers which helps to train the model the in detail the data is divided with the help of weights this weights helps to connect the neural network. The model is trained by all the folders contains different emotions like happy, sad, fear etc. With the help of this classified data we will train the model. We perform convolution over the input. Let $\mathrm{fk}$ be the filter having a kernel size $\mathrm{n} \times \mathrm{m}$ applied to the input connections on each neuron . The resulting output as follows:

$$
C\left(x_{u, v}\right)=\sum_{i=-\frac{n}{2}}^{\frac{n}{2}} \sum_{j=-\frac{m}{2}}^{\frac{m}{2}} f_{k}(i, j) x_{u-i, v-j}
$$

We use the above formula to calculate various representation of the input,we use many filters fk with can be applied on the various input. The filters fk were sharing the weights of neighboring neurons. Which helps to trained the model with lesser weights have to get standard Multilayer Perceptrons.We use a camera which helps to take the live images of the victim who is going to monitor for the recognition of expressions. We take the images and through the help of the openCV we run the live images with the help of the trained model and predicted the output and telecast the output simultaneously on the screen
In the output screen we have the live projection of the victim face and along with the state of the emotion of victim. We train the model by using DCNN and we connect camera to the system the patient is placed under the surveillance to take reading of the state of emotions of the victim and the facial expressions were telecast on the screen and we use algorithm to run on the real-world images of the victim and we find the state of the victim on the screen and if their any abnormal condition was shown on the screen then the person who monitors the victim inform the doctors to take of their situation and also used to record their state of mind in different situations

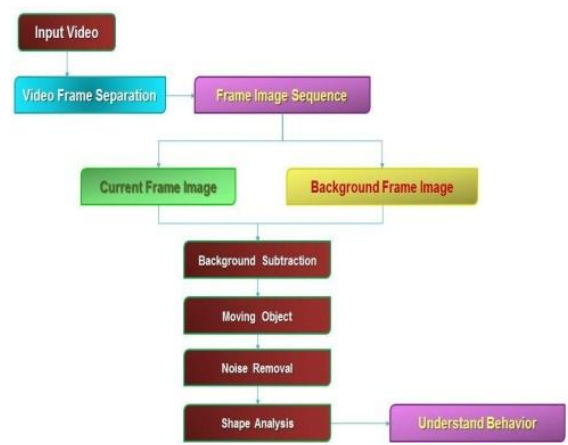

Fig 1 Workflow Diagram

\section{PERFORMANCE ANALYSIS \& RESULTS}

The input is an image which is taken from the camera where the victim of the autism disorder patients are placed and the image is run on the model which Is already trained by using Deep convolutional neural network(DCNN) this input images run continuously and they get the predictions from the train model and in the output screen we will we having the live state of emotion of the victim. This model gives the best accurate results comparing to other algorithms. In This we use openCV and tensorflow to run the program., The live object detection is only done with the help of OpenCV. The opencv helps to take live face detections to monitor the victim Facial expressions. The Tensorflow help to detect the emotion of the face object which is detecting through OpenCV, tensorflow is very power platform to handle powerful algorithm in deep learning, We run our algorithm in tensorflow and we use keras packages which is help to run the program in compact able machines

We evaluvated our algorithm based on the Accuracy,Sensitivity,Specificity and Flase positive rate(FPR)

\section{Acurracy $=\mathrm{Tr}+\mathrm{Tn} / \mathrm{N}$}

The above formulae we are using to calculate Acurracy and confusion matrix.We need to calculate confusion matrices to calculate which emotion is easier and which one difficult to distinguish. We also calculate the accuracy of the subject dependent class, The data divided into 6subsets and 4 facial expressions 
Table2: Confusion Matrix for Neural Network

\begin{tabular}{|l|l|l|l|l|}
\hline Emotions & Happy & Fear & Sad & neutral \\
\hline Happy & 420 & 0 & 6 & 9 \\
\hline Fear & 0 & 422 & 5 & 4 \\
\hline Sad & 4 & 1 & 426 & 15 \\
\hline Neutral & 2 & 0 & 8 & 425 \\
\hline
\end{tabular}

Table 3: Accuracy of subject dependent classification

\begin{tabular}{|c|l|l|}
\hline Subject & SVM & DCNN \\
\hline 1 & 0.70 & 0.75 \\
\hline 2 & 0.69 & 0.85 \\
\hline 3 & 0.60 & 0.67 \\
\hline 4 & 0.57 & 0.70 \\
\hline 5 & 0.71 & 0.78 \\
\hline 6 & 0.72 & 0.85 \\
\hline Average & 0.69 & 0.80 \\
\hline
\end{tabular}

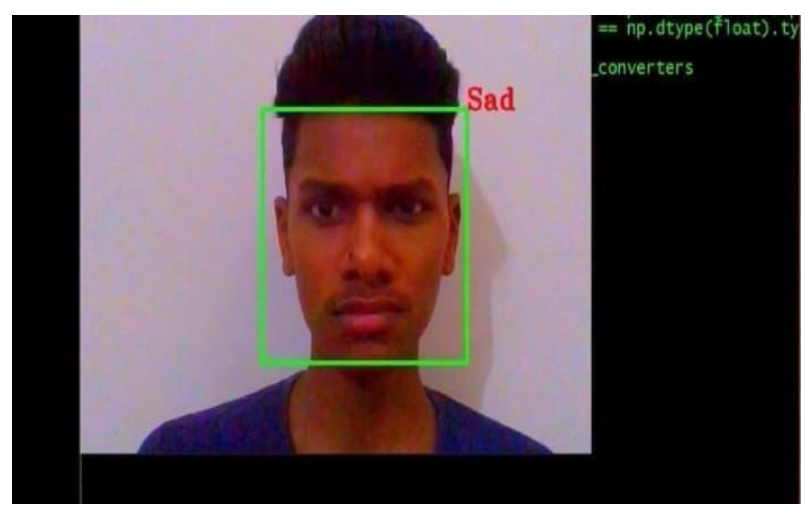

Fig 2 Emotion for Sadness

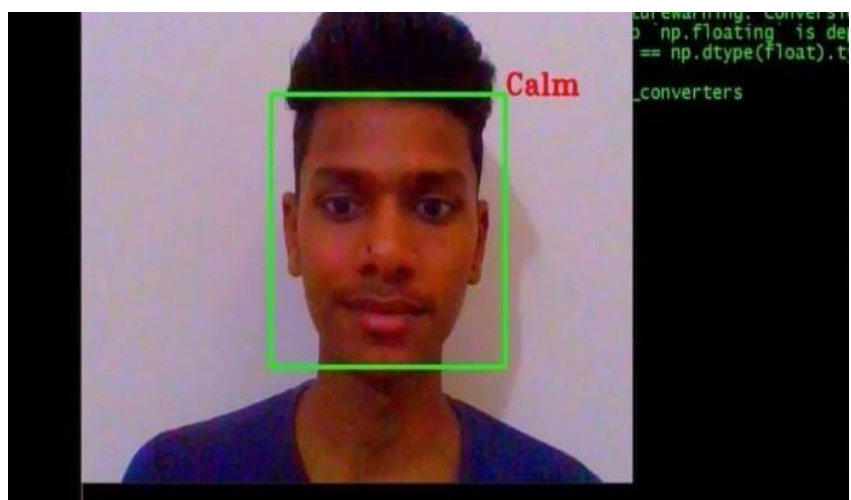

Fig 3 Emotion for Clam

\section{CONCLUSION}

This letter presented the Recognition of Facial Expressions for Autism Disorder people. We use camera to take live images of the Autism Disorder patient and we run the algorithm to find the state of mind of the patient and helps to take care of the patient health. This experiment help to take care of the people who doesn't have control over their emotions this help doctors to treat. Their patients easily. Now-a-days lot of people suffered with this kind of state because of their stress level and pressure which they can't have control on it.

\section{REFERENCES}

1. Veena Mayya, Radhika M. Pai*, Manohara Pai Automatic Facial Expression Recognition Using DCNN6th International
Conference On Advances In Computing \& Communications, ICACC 2016, 6-8 September 2016, Cochin, India

2. Paweł Tarnowski, Marcin Kołodziej, Andrzej Majkowski, Remigiusz J. Rak Emotion recognition using facial expressions International Conference on Computational Science, ICCS 2017, 12-14 June 2017, Zurich, Switzerland

3. Murthy.G.R.S. andR.S.Jadon. "Recognizing Facial Expressions Using Eigenspaces", in the proc of IEEE International Conference on Computational Intelligence and Multimedia Applications, Dec 2016,Sivakasi, Tamilnadu, India.

4. Aliaa A. A. Youssif, Wesam A.A.Asker "Automatic Facial Expression System Based on Geometric and Appearance Features", Published by Canadian Center of science and Education, computer and Information Science, march 2015.

5. Philipp Michel, Rana El Kaliouby " Real Time Facial Expression recognition in Video using Support Vector Machine" International Conference on Computational Science, ICCS 2017, June 2017, United Kingdom

6. Erhan, D., Szegedy, C., Toshev, A., Anguelov, D.. Scalable object detection using deep neural networks. In: Computer Vision and Pattern Recognition (CVPR), 2014 IEEE Conference on. 2014, p. 2155-2162. doi:10.1109/CVPR.2014.276.

7. Zhang, L., Tjondronegoro, D.. Facial expression recognition using facial movement features. IEEE Transactions on Affective Computing 2011;2(4):219-229. doi:10.1109/TAFFC.2016.

8. Dr.R.Subhashini and Milani.V, "IMPLEMENTING GEOGRAPHICAL INFORMATION SYSTEM TO PROVIDE EVIDENT SUPPORRT FOR CRIME ANALYSIS", International Conference on Intelligent Computing, Communication \& Convergence(ICCC-2014), Bhubaneswar, Odisha on 27th-28th December, 2014.

9. Anu, V. Maria, M. I. Deepika, and L. Mary Gladance. "Animal identification and data management using RFID technology." In International Confernce on Innovation Information in Computing Technologies, pp. 1-6. IEEE, 2015.

10. Subhashini, R., Sethuraman, R., Milani, V. "Providing Mother and Child Care Telemedicine Through Interactive Voice Response" Advances in Intelligent Systems and Computing Intelligent Computing, Communication and Devices Volume 308 of the series Advances in Intelligent Systems and Computing pp 771-778 